\title{
External stenting of vein grafts: Primum non nocere
}

\author{
Hadi Daood Toeg, MD, and Munir Boodhwani, MD
}

\author{
From the Division of Cardiac Surgery, University of Ottawa Heart Institute, Ottawa, Ontario, Canada. \\ Disclosures: Authors have nothing to disclose with regard to commercial support. \\ Received for publication June 18, 2015; accepted for publication June 23, 2015; available ahead of print July 26, \\ 2015. \\ Address for reprints: Munir Boodhwani, MD, Division of Cardiac Surgery, University of Ottawa Heart Institute, \\ H3404 - 40 Ruskin St, Ottawa, Ontario, Canada (E-mail: mboodhwani@ottawaheart.ca). \\ J Thorac Cardiovasc Surg 2015;150:774-5 \\ $0022-5223 / \$ 36.00$ \\ Copyright $(c) 2015$ by The American Association for Thoracic Surgery \\ http://dx.doi.org/10.1016/j.jtcvs.2015.06.056
}

The Achilles heel of coronary artery bypass graft (CABG) surgery is saphenous vein graft (SVG) endothelial damage and intimal hyperplasia, culminating in atherosclerosis and early-to-late graft failure. ${ }^{1}$ Contemporary SVG patency rates at 1 year after $\mathrm{CABG}$ can reach $80 \%$ to $89 \%$. However, at 10 years, only one half of SVGs are patent; the remainder demonstrate varying levels of atherosclerosis. Thus, total arterial grafting is the optimal strategy to improve longterm patency and outcome.

Nevertheless, SVGs continue to play an important role in the treatment of elderly patients and those with unavailable or prohibitive risk with arterial conduit harvest. A potential approach to improving SVG patency includes external SVG stenting; materials used for this purpose include porous polyester, nitinol mesh, ${ }^{2}$ metallic biocompound, and cobalt-chromium stents. ${ }^{3}$ External stenting is believed to improve SVG patency and reduce intimal hyperplasia, by: (1) imposing SVG symmetry, leading to laminar flow and reduction in aberrant hemodynamic forces; and (2) creating a protective environment to promote formation of a microvascular-rich neoadventitia. ${ }^{3}$ However, despite promising preclinical studies with external stenting, ${ }^{4} \mathrm{Re}-$ scigno and colleagues ${ }^{2}$ demonstrated very poor 12 -month SVG occlusion rates of $62 \%$ with nitinol mesh stents. ${ }^{2}$

Taggart and colleagues ${ }^{5}$ randomized 30 patients to either an SVG external stent or not, and reported their 12-month results after angiography and intravascular ultrasonography. Significantly more SVG failures occurred in the stented group compared with the nonstented group when veins were grafted to the right coronary territory (46.2\% vs $13.4 \% ; P=.01)$, whereas the opposite was seen when stented SVGs were grafted to the left territory $(17.6 \%$ vs $27.5 \% ; P=.02)$. The extent of intimal hyperplasia assessed by intravascular ultrasonography was marginally less prevalent in the stented group, compared with controls $(P=.04)$. Although this study demonstrates some potential in reducing intimal hyperplasia with external SVG stenting, the high SVG occlusion rate overall, particularly in the right coronary territory, is concerning.

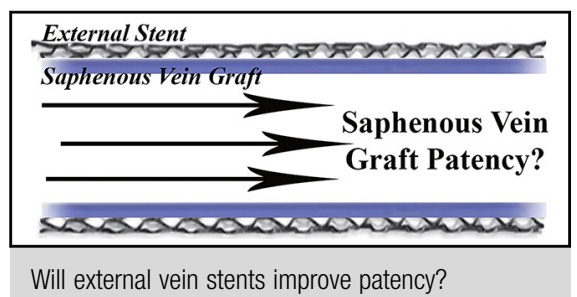

Central Message

Measuring stented and nonstented SVGs, using computational fluid dynamics modeling, in the setting of CABG, indicates that oscillatory shear index is correlated with intimal hyperplasia.

See Article page 871

Meirson and colleagues ${ }^{6}$ present a post hoc analysis in an effort to correlate hemodynamic factors with intimal hyperplasia. The authors performed geometric reconstruction of all SVGs (stented and nonstented), and generated 3-dimensional models derived from finite-element modeling. The hemodynamic parameters of interest included time-averaged wall shear stress, representing SVG tangential frictional forces, and oscillatory shear index, representing the degree of deviation of wall shear stress flow direction. The mean time-averaged wall shear stress did not differ significantly between groups, whereas the mean oscillatory shear index was significantly lower in the stented group $(P=.009)$. Finally, the mean oscillatory shear index values in all groups were correlated with diffuse intimal hyperplasia as measured by intravascular ultrasonography $(P=.01)$.

This study presents computational fluid dynamics modeling as another potential tool in understanding progression of SVG disease. This correlation needs to be recapitulated in other studies, using larger sample sizes and possibly other imaging modalities for validation. If validated, these parameters may be utilized to explore new therapies that optimize fluid dynamics in vein grafts and potentially improve disease-free durability.

Several study limitations need to be considered when interpreting the data from Meirson and colleagues. ${ }^{6}$ First, the 3-D finite element model was derived from 2-D images without baseline hemodynamic parameters, which may be better reproduced with 3-D computed tomography angiography. ${ }^{7}$ Second, this study had a small sample size for intravascular ultrasonography and hemodynamic measurements, as $23 \%$ of all SVGs were occluded. Third, the 
presence of mild varicosities or valves was not incorporated into the models. Last, in addition to the methodologic limitations, a potential conflict of interest is inherent in this industry-sponsored trial.

In summary, this group is commended for devising another measurement tool in assessing SVG disease in the setting of CABG. Although this study has demonstrated a direct correlation between 1 hemodynamic parameteroscillatory shear index (blood velocity oscillation and reciprocation) - and intimal hyperplasia, the clinical significance of this finding remains unclear, and future studies are required to validate this finding. Although data from this study suggest a reduction in intimal hyperplasia with external SVG supports, ${ }^{3}$ caution must be exercised in externally stenting SVGs, particularly in the right territory, because of the increased risk of graft occlusion. ${ }^{2,5,6}$ After all, a slightly diseased SVG is still better than an occluded one.

\section{References}

1. Kim FY, Marhefka G, Ruggiero NJ, Adams S, Whellan DJ. Saphenous vein graf disease: review of pathophysiology, prevention, and treatment. Cardiol Rev. 2013; 21:101-9.

2. Rescigno G, Aratari C, Matteucci SM, Parisi R, Gironi G, Schicchi N, et al. Saphenous vein graft wrapping by nitinol mesh: a word of caution. Thorac Cardiovasc Surg. 2015;63:292-7.

3. Jeremy JY, Gadsdon P, Shukla N, Vijayan V, Wyatt M, Newby AC, et al. On the biology of saphenous vein grafts fitted with external synthetic sheaths and stents. Biomaterials. 2007:28:895-908.

4. Angelini GD, Lloyd C, Bush R, Johnson J, Newby AC. An external, oversized, porous polyester stent reduces vein graft neointima formation, cholesterol concentration, and vascular cell adhesion molecule 1 expression in cholesterol-fed pigs. $J$ Thorac Cardiovasc Surg. 2002;124:950-6.

5. Taggart DP, Ben Gal Y, Lees B, Patel N, Webb C, Rehman SM, et al. A randomized trial of external stenting for saphenous vein grafts in coronary artery bypass grafting. Ann Thorac Surg. 2015;99:2039-45.

6. Meirson T, Orion E, Di Mario C, Webb C, Patel N, Channon KM, et al. Flow patterns in externally stented saphenous vein grafts as related to the development of intimal hyperplasia. J Thorac Cardiovasc Surg. 2015;150:871-9.

7. Morganti S, Valentini A, Favalli V, Serio A, Gambarin FI, Vella D, et al. Aortic root 3D parametric morphological model from 2D-echo images. Comput Biol Med. 2013;43:2196-204. 